Introduction: Neopterin concentrations usually correlate with immune activation and are significant predictors of chronic infections, various types of cancer and CVD. In adults, an association between increased BMI and neopterin concentrations was observed, but although there is evidence for a proinflammatory state accompanied by impaired vascular endothelial function in childhood and adolescence, such data are still scarce.

Method: We investigated serum neopterin concentrations (ELISA, BRAHMS, Hennigsdorf, Germany) as well as tryptophan metabolism (HPLC) in 356 overweight and obese (aged $11.3(\mathrm{sD} 2.97)$ years; $\mathrm{f}=\mathrm{m}$ ), otherwise healthy children and thirty-two non-obese controls.

Results: BMI differed significantly between obese and nonobese probands (28 (SD 5.64) v. $18(\mathrm{SD} 2 \cdot 19) \mathrm{kg} / \mathrm{m}^{2}, U=9 \cdot 24$,
$P<0000 \cdot 1$, Mann-Whitney $U$-test). Neopterin concentrations were similar in both groups, although low as compared with reported adult data. By contrast, tryptophan concentrations were significantly lower in the obese $(74 \cdot 7+18 \cdot 8 \mathrm{qmol} / \mathrm{l}) v$. non-obese subjects $(80 \cdot 7+12 \cdot 7 \mathrm{qmol} / 1, U=2 \cdot 384, P=0 \cdot 017)$, while there was no difference in kynurenine concentrations.

Conclusions: Obesity in juveniles is not associated with increased neopterin concentrations suggesting that obesity at least in the early course of the disease does not lead directly to Th1-type immune activation and associated CVD. Only in the later course a switch to Th-1 type immune activation and associated CVD may take place. Chronic infections or other cofactors might be important to trigger cytokine production and elevated neopterin concentrations at the later stage.

\title{
24 - May acanthosis nigricans be a predictor of insulin resistance in obese adolescents?
}

\author{
R Gomes ${ }^{1}, M$ Pinto $^{1}, \mathrm{P}$ Nogueira ${ }^{2}$ and H Fonseca ${ }^{1}$ \\ 'Department of Paediatrics, Paediatric Obesity Clinic, Hospital de Santa Maria, Lisboa, Portugal: ${ }^{2}$ Epidemiology \\ Division, Instituto de Medicina Preventiva, Faculdade de Medicina de Lisboa, Portugal
}

Introduction: Acanthosis nigricans (AN) is a condition characterized by hyperpigmented, papillomatous and hyperkeratotic skin and is associated with obesity and impaired glucose tolerance. The purpose of the present study was to explore a potential association between $\mathrm{BMI}$, insulin resistance and $\mathrm{AN}$.

Method: A retrospective review of the files of obese adolescents at their first visit to the clinic was conducted. The sample was divided considering the presence or absence of AN. Age, BMI (Z-score) and fast glucose and insulin blood levels were compared between the two groups using a bivariate analysis. The insulin resistance index was calculated based on the homeostasis model assessment (HOMA).

Results: Data from 116 obese adolescents, forty-seven males, median age 13 years, was collected. AN was found in fifty-three subjects: cervical location (21), axilar location (3) and both (15). In fourteen adolescents the location was not specified. Mean BMI Z-score was 3.67 within the AN group and 3.03 among those without AN. A mean HOMA of 4.02 was found in the AN group compared with $2 \cdot 72$ among those without AN. A significant association was found between HOMA $(P=0 \cdot 009)$, BMI $Z$-score $(P=0 \cdot 01)$ and $\mathrm{AN}$.

Conclusions: According to the results, BMI Z-score and HOMA are significantly higher once $\mathrm{AN}$ is present. Therefore, the presence of AN should be routinely assessed in obese adolescents. Future research should focus on the association between the improvement of both BMI and HOMA and the regression of AN.

\section{5 - A case of severe obesity with metabolic syndrome in an adolescent: diagnosis and management}

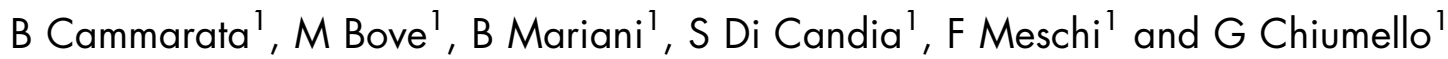 \\ 'Department of Paediatrics, Endocrine Unit, San Raffaele Scientific Institute, Vita Salute San Raffaele University, \\ Milan, Italy
}

